\title{
ALMOST-PRIME VALUES OF REDUCIBLE POLYNOMIALS AT PRIME ARGUMENTS
}

\author{
C. S. FRANZE AND P. H. KAO
}

\begin{abstract}
We adopt A. J. Irving's sieve method to study the almost-prime values produced by products of irreducible polynomials evaluated at prime arguments. This generalizes the previous results of Irving and Kao, who separately examined the almost-prime values of a single irreducible polynomial evaluated at prime arguments.
\end{abstract}

\section{INTRODUCTION}

In this paper, we adopt a sieve method developed by A. J. Irving in [7] to prove

Theorem 1. Let $H(n)=h_{1}(n) \cdots h_{g}(n)$, where $h_{i}$ are distinct irreducible polynomials each with integer coefficients and $\operatorname{deg} h_{i}=k$ for all $i=1, \ldots, g$. Suppose that

$$
\#\{a(\bmod p):(a, p)=1 \text { and } H(a) \equiv 0(\bmod p)\}<p-1 .
$$

Then, for sufficiently large $x$, there exists a natural number $r$ such that

$$
\sum_{\substack{x<p \leqslant 2 x \\ \Omega(H(p)) \leqslant r}} 1 \gg \frac{x}{\log ^{g+1} x} .
$$

If $g \geqslant 2$ and $k$ is sufficiently large, we may select an $r$ of the form

$$
r=g k+c_{1} g^{3 / 2} k^{1 / 2}+c_{2} g^{2}+O(g \log g k),
$$

where $c_{1}$ and $c_{2}$ are $O(1)$. Explicit admissible values of $r$ for small $g$ and $k$ are given below.

\begin{tabular}{|c|r|r|r|r|r|r|r|r|r|r|r|r|r|r|}
\hline$g \backslash k$ & 1 & 2 & 3 & 4 & 5 & 6 & 7 & 8 & 9 & 10 & 11 & 12 & 13 & 14 \\
\hline \hline 2 & - & - & 15 & 18 & 21 & 23 & 26 & 29 & 31 & 33 & 36 & 38 & 40 & 43 \\
\hline 3 & - & - & - & 30 & 35 & 39 & 43 & 47 & 51 & 55 & 59 & 62 & 66 & 70 \\
\hline 4 & - & - & - & 43 & 50 & 56 & 63 & 68 & 74 & 79 & 85 & 90 & 95 & 100 \\
\hline
\end{tabular}

TABLE 1. Admissible values for $r$ using Irving's sieve method

The case $g=1$ was first investigated by H.-E. Richert in 1969 [9], who showed that for each $k \geqslant 1, r=2 k+1$ is an admissible choice. Virtually no progress was made until Irving's work in 2015 [7], which showed that one could take an $r$ of the form $r=k+O(\log k)$. Explicit bounds for the $O$-term, as well as explicit values for $r$ when $k$ is small, are available in [7] and [8].

The more general case where $g \geqslant 2$ is studied in the book by Halberstam and Richert in 1974 [6], who showed that one could select an $r$ of the form

$$
r=2 g k+O(g \log g k) \text {. }
$$


Their method was refined in the book by Diamond and Halberstam [4], which offers the admissible $r$ described below in Table 2 (see [4, pp.149-150]). However, their admissible $r$ exhibit the same asymptotic behavior described in (3). Therefore, the results of Theorem 1 represent an improvement when $k \gg g$.

\begin{tabular}{|c|r|r|r|r|r|r|r|r|r|r|r|r|r|r|}
\hline$g \backslash k$ & 1 & 2 & 3 & 4 & 5 & 6 & 7 & 8 & 9 & 10 & 11 & 12 & 13 & 14 \\
\hline \hline 2 & 7 & 11 & 16 & 20 & 24 & 28 & 32 & 36 & 40 & 44 & 48 & 52 & 56 & 60 \\
\hline 3 & 12 & 19 & 25 & 32 & 38 & 44 & 50 & 56 & 62 & 69 & 75 & 81 & 87 & 93 \\
\hline 4 & 17 & 27 & 35 & 44 & 52 & 61 & 69 & 77 & 86 & 94 & 102 & 110 & 118 & 126 \\
\hline
\end{tabular}

TABLE 2. Classical admissible values for $r$

Irving's innovation was to combine a linear (one-dimensional) sieve with a two-dimensional sieve that permits a level of distribution beyond that which is available using the Bombieri-Vinogradov theorem. We adopt this novel idea to the relevant $g$ - and $g+1$-dimensional sieves used for the more general polynomial sequence, $H$, considered here. The sifting functions $F_{g}$ and $f_{g}$ are, however, more difficult to work with for $g \geqslant 2$.

\section{Main Sieve Setup}

Here, we adopt some standard sieve notation. Setting $P(z)=\prod_{p<z} p$, we require bounds on

$$
S(A, z)=\#\{n \in A:(n, P(z))=1\} .
$$

The sequence that we are going to sieve is

$$
A=\{H(p): x<p \leqslant 2 x\} .
$$

Using the prime number theorem, we note that the cardinality $|A| \sim X$, where

$$
X=\operatorname{li} x
$$

Letting $A_{d}=\{n \in A: n \equiv 0(d)\}$, it is straightforward (e.g. see [4, pp.131-132]) to show that

$$
\left|A_{d}\right|=\frac{\rho_{1}(d)}{\phi(d)} X+r_{A}(d)
$$

where

$$
\rho_{1}(d):=\#\{a(\bmod d): 1 \leqslant a \leqslant d \text { and }(a, d)=1 \text { and } H(a) \equiv 0(\bmod d)\},
$$

and the remainder term, $r_{A}(d)$, is bounded by

$$
\left|r_{A}(d)\right| \leqslant \rho(d) E(x, d)+\rho(d)
$$

where

$$
\rho(d):=\#\{a(\bmod d): 1 \leqslant a \leqslant d \text { and } H(a) \equiv 0(\bmod d)\}
$$

and

$$
E(x, d)=\max _{\substack{1 \leqslant m \leqslant d \\(m, d)=1}}\left|\pi(x, d, m)-\frac{\operatorname{li} x}{\phi(d)}\right| .
$$


The sieve dimension is $g$ since the density function $\rho_{1}(d) / \phi(d)$ appearing in (6) satisfies

$$
\sum_{p \leqslant x} \frac{\rho_{1}(p)}{\phi(p)} \log p=g \log x+O(1) .
$$

This follows from Proposition 10.1 of [4], which gives

$$
\sum_{p \leqslant x} \frac{\rho_{1}(p)}{p} \log p=g \log x+O(1),
$$

since

$$
\sum_{p \leqslant x}\left(\frac{\rho_{1}(p)}{\phi(p)}-\frac{\rho_{1}(p)}{p}\right) \log p \ll \sum_{p \leqslant x} \frac{\rho_{1}(p)}{p^{2}} \log p \ll_{H} \sum_{p \leqslant x} \frac{\log p}{p^{2}} \ll 1,
$$

where we used $\rho_{1}(p) \leqslant \rho(p) \leqslant \operatorname{deg}(H)$.

As a consequence of (7), the product

$$
V(z):=\prod_{p<z}\left(1-\frac{\rho_{1}(p)}{\phi(p)}\right) \gg(\log z)^{-g} .
$$

Finally, we note that the Bombieri-Vinogradov theorem implies that for any $\tau_{1} \leqslant \frac{1}{2}$,

$$
\sum_{\substack{d s q u a r e f r e e \\ d<X^{\tau_{1}}(\log X)^{-B}}} 4^{\omega(d)}\left|r_{A}(d)\right| \ll \frac{X}{(\log X)^{g+1}},
$$

for a suitably large value of $B$ (e.g. see [6, Lemma 3.5 on p.115, and p. 288]). The parameter $\tau_{1}$ is called the level of distribution.

\section{An Auxiliary Sieve}

The main difference between Irving's approach, adopted here, and the classical one is the introduction of an auxiliary upper bound sieve for the sequence $A_{p}$, where $p$ is a prime $z \leqslant p<y$. Recall from (4) that

$$
S\left(A_{p}, z\right)=\sum_{\substack{x<q \leqslant 2 x \\ q \text { prime } \\ H(q) \equiv 0(p) \\(H(q), P(z))=1}} 1 .
$$

If $z<x$, then for any prime $q>x$ we plainly have $(q, P(z))=1$. Therefore,

$$
S\left(A_{p}, z\right)=\sum_{\substack{x<q \leqslant 2 x \\
q \text { prime } \\
H(q) \equiv 0(p) \\
(q H(q), P(z))=1}} 1 \leqslant \sum_{\begin{array}{c}
x<n \leqslant 2 x \\
H(n) \equiv 0(p) \\
(n H(n), P(z))=1
\end{array}} 1=S\left(A^{\prime}, z\right),
$$

where

$$
A^{\prime}=\{n H(n): x<n \leqslant 2 x, p \mid H(n)\} .
$$

Although the upper bound available for $S\left(A^{\prime}, z\right)$ is worse than that for $S\left(A_{p}, z\right)$, a larger level of distribution is available to us for $A^{\prime}$, which involves integer arguments rather than primes. In this case, the cardinality $\left|A^{\prime}\right| \sim X^{\prime}$, where

$$
X^{\prime}=\frac{\rho_{1}(p)}{p} x
$$


and, using the Chinese remainder theorem, we observe that

$$
\left|A_{d}^{\prime}\right|=\frac{\rho_{2}(d)}{d} X^{\prime}+r_{A^{\prime}}(d)
$$

where

$$
\rho_{2}(d):=\#\{a(\bmod d): a H(a) \equiv 0(\bmod d)\},
$$

and the remainder term, $r_{A^{\prime}}(d)$, is bounded by

$$
\left|r_{A^{\prime}}(d)\right| \leqslant \rho_{1}(p) \rho_{2}(d)
$$

for $d \mid P(z)$ and $p \geqslant z$ large enough to ensure that $p \nmid H(0)$ (see proof of Lemma 4.2 in [7]). The sieve dimension is $g+1$ in this case since the density function $\rho_{2}(d) / d$ appearing in (12) satisfies

$$
\sum_{p \leqslant x} \frac{\rho_{2}(p)}{p} \log p=(g+1) \log x+O(1)
$$

owing to the fact that $\rho_{2}(p)=\rho_{1}(p)+1$. As a consequence of (14), we have

$$
V^{\prime}(z):=\prod_{p<z}\left(1-\frac{\rho_{2}(p)}{p}\right) \gg(\log z)^{-(g+1)} .
$$

More precisely, using Mertens' product formula,

$$
V^{\prime}(z)=\prod_{p<z}\left(1-\frac{1}{p}\right)\left(1-\frac{\rho_{1}(p)}{\phi(p)}\right) \sim \frac{e^{-\gamma}}{\log z} V(z) .
$$

Using (5), we note that

$$
x \sim X \log X
$$

and therefore,

$$
X^{\prime} \sim \frac{\rho_{1}(p)}{p} X \log X
$$

In contrast to (10), upon setting $z=X^{1 / v}$, a small power of $X$, we see that for any $\tau_{2} \leqslant 1$,

$$
\sum_{d \mid P(z)} 4^{\omega(d)}\left|r_{A^{\prime}}(d)\right|=o\left(X^{\prime} V^{\prime}(z)\right)
$$

for a suitably large $B^{\prime}$. This is easily obtained using (13) and (15) so that

$$
\sum_{\substack{d \mid P(z) \\ \tau_{2}(\log X)^{-B^{\prime}}}} 4^{\omega(d)}\left|r_{A^{\prime}}(d)\right| \leqslant \frac{\rho_{1}(p)}{p} X^{\tau_{2}}(\log X)^{-B^{\prime}} \sum_{d \mid P(z)} \frac{4^{\omega(d)} \rho_{2}(d)}{d} .
$$

Proceeding in the manner of the proof of Lemma 4.3 in [4], we conclude that this is

$$
X^{\prime}\left(\frac{X^{\tau_{2}}}{x}\right)(\log X)^{-B^{\prime}} \prod_{p<z}\left(1+\frac{4 \rho_{2}(p)}{p}\right) \ll X^{\prime}\left(\frac{\operatorname{li} x}{x}\right)(\log X)^{-B^{\prime}} V^{\prime}(z)^{-4}=o\left(X^{\prime} V^{\prime}(z)\right),
$$

for a suitably large $B^{\prime}$. 


\section{Diamond-Halberstam-Richert Sieve}

We will employ the Diamond-Halberstam-Richert (DHR) sieve to estimate the number of survivors, $S(A, z), S\left(A_{p}, z\right)$, and $S\left(A^{\prime}, z\right)$. Recall from Theorem 9.1 of [4] that for any $2 \leqslant z \leqslant y$,

$$
S(A, z) \leqslant X V(z)\left(F_{g}\left(\frac{\log y}{\log z}\right)+O\left(\frac{(\log \log y)^{2}}{(\log y)^{1 /(2 g+2)}}\right)\right)+2 \sum_{\substack{m \mid P(z) \\ m<y}} 4^{\omega(m)}\left|r_{A}(m)\right|,
$$

and,

$$
S(A, z) \geqslant X V(z)\left(f_{g}\left(\frac{\log y}{\log z}\right)-O\left(\frac{(\log \log y)^{2}}{(\log y)^{1 /(2 g+2)}}\right)\right)-2 \sum_{\substack{m \mid P(z) \\ m<y}} 4^{\omega(m)}\left|r_{A}(m)\right| .
$$

The functions $F_{g}$ and $f_{g}$ are defined by the unique solutions to the differential-delay equations

$$
\begin{aligned}
\left(u^{g} F_{g}(u)\right)^{\prime} & =g u^{g-1} f_{g}(u-1), & & u>\alpha_{g} \\
\left(u^{g} f_{g}(u)\right)^{\prime} & =g u^{g-1} F_{g}(u-1), & & u>\beta_{g},
\end{aligned}
$$

with initial conditions

$$
\begin{aligned}
& F_{g}(u)=\frac{1}{\sigma_{g}(u)}, \quad 0<u \leqslant \alpha_{g}, \\
& f_{g}(u)=0, \quad 0<u \leqslant \beta_{g},
\end{aligned}
$$

where $\sigma_{g}$ is the Ankeny-Onishi function, and

$$
\alpha_{1}=\beta_{1}=2 \text { and } \alpha_{g}>\beta_{g}>2 \text { for } g>1 .
$$

We suppose here that $g$ is a positive integer, and remark that Booker and Browning [2] have recently compiled a list of values for $\alpha_{g}$ and $\beta_{g}$ for $g \leqslant 50$. The sifting limit $\beta_{g}$ satisfies $\beta_{g} \lesssim c g$, where $c \approx 2.445$ (see [3, Theorem 2], and [1]). The functions $F_{g}$ and $f_{g}$ satisfy

$$
F_{g}(u)=1+O\left(e^{-u}\right), \quad f_{g}(u)=1+O\left(e^{-u}\right),
$$

and $F_{g}$ decreases monotonically, while $f_{g}$ increases monotonically on $(0, \infty)$. In fact, Diamond and Halberstam establish in [4, Lemma 6.2] that for $1 \leqslant u_{1}<u_{2}$,

$$
0 \leqslant F_{g}\left(u_{1}\right)-F_{g}\left(u_{2}\right) \leqslant \frac{u_{2}-u_{1}}{u_{1}} \cdot \frac{g}{\sigma_{g}(1)},
$$

and

$$
0 \leqslant f_{g}\left(u_{2}\right)-f_{g}\left(u_{1}\right) \leqslant \frac{u_{2}-u_{1}}{u_{1}} \cdot \frac{g}{\sigma_{g}(1)}
$$

\section{RICHERT WEIGHTS}

The aforementioned DHR sieve is enhanced by incorporating certain weights introduced by Richert [9]. The arithmetic significance of these weights are summarized in the lemma below.

Lemma 5.1. Suppose $y=X^{1 / u}, z=X^{1 / v}$, and $0<\frac{1}{v}<\frac{1}{u}<\tau_{2} \leqslant 1$. Let $r$ be a natural number such that $r+1>g k u$, and define $\eta:=r+1-g k u$. Then for $x$ sufficiently large,

$$
\sum_{\substack{n \in A \\ \Omega(n) \leqslant r \\(n, P(z))=1}} 1 \geqslant \frac{1}{r+1} W(A)-o(X V(z))
$$


where

$$
W(A):=\sum_{\substack{n \in A \\(n, P(z))=1}}\left(\eta-\sum_{\substack{z \leqslant p<y \\ p \mid n}}\left(1-\frac{\log p}{\log y}\right)\right) .
$$

Thus, if we can show that the weighted sum $W(A)$ remains large even as $x$ grows large, say for example $W(A) \gg X V(z)$, then we succeed in demonstrating the abundance of elements $n \in A$ which contain at most $r$ prime factors. The proof of this lemma is contained in [4, pp.140-141]. We briefly reproduce it here for completeness.

Proof. We begin by observing that the number of elements $n \in A$ that are divisible by $p^{2}$ for a $z \leqslant p<y$ is negligible. More specifically,

$$
\sum_{z \leqslant p<y}\left|A_{p^{2}}\right| \ll \sum_{z \leqslant p<y} \rho\left(p^{2}\right)\left(\frac{x}{p^{2}}+O(1)\right) \ll_{H} \frac{x}{z}+y=o(X V(z)),
$$

since $\rho\left(p^{2}\right) \leqslant \operatorname{deg}(H) D^{2}$, where $D$ is the discriminant of $H$ [6, p. 260]. Therefore, we have

$$
W(A)=W\left(A^{*}\right)+o(X V(z))
$$

where

$$
A^{*}:=A \backslash \bigcup_{z \leqslant p<y} A_{p^{2}} .
$$

If an $n \in A^{*}$ contains a repeated prime factor $p$, then $p \geqslant y$, and so

$$
\sum_{\substack{z \leqslant p<y \\ p \mid n}}\left(1-\frac{\log p}{\log y}\right) \geqslant \sum_{\substack{p \geqslant y \\ p \mid n}}^{*}\left(1-\frac{\log p}{\log y}\right)=\Omega(n)-\frac{\log |n|}{\log y} \geqslant \Omega(n)-\frac{\log X^{g k}}{\log X^{1 / u}}
$$

where $\sum^{*}$ denotes summation over the appropriate multiplicity. It follows from (27) and (29) that

$$
W\left(A^{*}\right) \leqslant \sum_{\substack{n \in A^{*} \\(n, P(z))=1}}(r+1-\Omega(n)) \leqslant \sum_{\substack{n \in A^{*} \\ \Omega(n) \leqslant r \\(n, P(z))=1}}(r+1) .
$$

Combining this inequality with (28) finishes the proof of the lemma since

$$
\sum_{\substack{n \in A \\ \Omega(n) \leqslant r \\(n, P(z))=1}} 1 \geqslant \sum_{\substack{n \in A^{*} \\ \Omega(n) \leqslant r \\(n, P(z))=1}} 1 \geqslant \frac{1}{r+1} W(A)-o(X V(z))
$$

The observant reader may note that $g k$ should be replaced with $g k+\varepsilon$ in (29) since

$$
\max _{n \in A^{*}}|n| \leqslant X^{g k+\varepsilon}
$$

for $x$ sufficiently large. The presence of this $\varepsilon$, however, makes little difference in the final analysis. 


\section{Approximating the Weighted Sum}

In this section, we turn our attention to approximating the weighted sum, $W(A)$, by integrals. Recall that $z=X^{1 / v}$ and $y=X^{1 / u}$. Letting $s \in(z, y)$, say $s=X^{1 / w}$, we have

$$
W(A)=\eta S(A, z)-\left(S_{1}+S_{2}\right)
$$

where

$$
S_{1}:=\sum_{z \leqslant p<s}\left(1-\frac{\log p}{\log y}\right) S\left(A_{p}, z\right)
$$

and,

$$
S_{2}:=\sum_{s \leqslant p<y}\left(1-\frac{\log p}{\log y}\right) S\left(A_{p}, z\right) .
$$

For $S(A, z)$ and $S_{1}$, we invoke the Bombieri-Vinogradov theorem in (10) for the underlying $g$ dimensional sieve. However, for $S_{2}$, we will swap $S\left(A_{p}, z\right)$ for $S\left(A^{\prime}, z\right)$, where we can instead make use of (18) for the underlying $(g+1)$-dimensional sieve. For readers who wish to skip ahead, we are ultimately lead to an integral form for $W(A)$ stated below in Lemma 6.4. The following three lemmas provide the necessary bounds for $S(A, z), S_{1}$, and $S_{2}$.

Lemma 6.1. Let $z=X^{1 / v}$, and $0<\frac{1}{v}<\tau_{1} \leqslant \frac{1}{2}$. Then

$$
S(A, z) \geqslant X V(z)\left\{f_{g}\left(\tau_{1} v\right)-o(1)\right\} .
$$

Proof. Letting $y=X^{\tau_{1}}(\log X)^{-B}, X=\operatorname{li} x$, we conclude at once from (9), (10), and (20) that

$$
S(A, z) \geqslant X V(z)\left\{f_{g}\left(\tau_{1} v-B v \frac{\log \log X}{\log X}\right)-O\left(\frac{(\log \log X)^{2}}{(\log X)^{1 /(2 g+2)}}\right)\right\}-o(X V(z)) .
$$

Finally, equation (25) allows us to perturb the argument of $f_{g}$ at a small expense, so that

$$
f_{g}\left(\tau_{1} v-B v \frac{\log \log X}{\log X}\right) \geqslant f_{g}\left(\tau_{1} v\right)-O\left(\frac{\log \log X}{\log X}\right) .
$$

Lemma 6.2. Let $z=X^{1 / v}, s=X^{1 / w}$, and $y=X^{1 / u}$ where $0<\frac{1}{v}<\frac{1}{w}<\tau_{1} \leqslant \frac{1}{2}<\frac{1}{u}$. Then

$$
S_{1} \leqslant X V(z) g\left\{\int_{w}^{v}\left(1-\frac{u}{t}\right) F_{g}\left(v\left(\tau_{1}-\frac{1}{t}\right)\right) \frac{d t}{t}+o(1)\right\} .
$$

Proof. We apply the $g$-dimensional upper bound DHR sieve in (19) to $S\left(A_{p}, z\right)$ with level of distribution $X^{\tau_{1}} / p$. Letting $z=X^{1 / v}, y=X^{\tau_{1}}(\log X)^{-B} / p$ in (19), we have

$$
\frac{(\log \log y)^{2}}{(\log y)^{1 /(2 g+2)}} \ll \frac{(\log \log X)^{2}}{\left(\log \left(X^{\tau_{1}}(\log X)^{-B} / p\right)\right)^{1 /(2 g+2)}} \ll \frac{(\log \log X)^{2}}{\left(\log \left(X^{\tau_{1}-\frac{1}{w}}(\log X)^{-B}\right)\right)^{1 /(2 g+2)}},
$$

and so,

$$
S\left(A_{p}, z\right) \leqslant \frac{\rho_{1}(p)}{\phi(p)} X V(z)\left(F_{g}\left(\frac{\log \left(X^{\tau_{1}}(\log X)^{-B} / p\right)}{\log X^{1 / v}}\right)+o(1)\right)+2 \sum_{m \in \mathcal{M}_{p}} 4^{\omega(m)}\left|r_{A_{p}}(m)\right|,
$$

where

$$
\mathcal{M}_{p}:=\left\{m \mid P(z): m<X^{\tau_{1}}(\log X)^{-B} / p\right\}
$$


Applying (24) to perturb the argument of $F_{g}$ at a small expense, we have

$$
S\left(A_{p}, z\right) \leqslant \frac{\rho_{1}(p)}{\phi(p)} X V(z)\left(F_{g}\left(\tau_{1} v-v \frac{\log p}{\log X}\right)+o(1)\right)+2 \sum_{m \in \mathcal{M}_{p}} 4^{\omega(m)}\left|r_{A}(p m)\right| .
$$

Now, summing over $p$ in $S_{1}$, we have

$$
S_{1} \leqslant X V(z) \sum_{z \leqslant p<s}\left(1-\frac{\log p}{\log y}\right) \frac{\rho_{1}(p)}{\phi(p)}\left(F_{g}\left(\tau_{1} v-v \frac{\log p}{\log X}\right)+o(1)\right)+o(X V(z)),
$$

since, by the Bombieri-Vinogradov in 10,

$$
\sum_{z \leqslant p<s} \sum_{m \in \mathcal{M}_{p}} 4^{\omega(m)}\left|r_{A}(p m)\right| \ll \sum_{\substack{n<X^{\tau}(\log X)^{-B} \\ n \text { squarefree }}} 4^{\omega(n)}\left|r_{A}(n)\right|=o(X V(z)) .
$$

Using (7), and recalling that $z=X^{1 / v}$, and $s=X^{1 / w}$, we find that

$$
\sum_{z \leqslant p<s} \frac{\rho_{1}(p)}{\phi(p)} \ll g \log \left(\frac{\log s}{\log z}\right) \ll g \log \frac{v}{w} \ll 1 .
$$

Therefore, distributing the sum in (30) gives

$$
S_{1} \leqslant X V(z)\left(\sum_{z \leqslant p<s}\left(1-\frac{\log p}{\log y}\right) \frac{\rho_{1}(p)}{\phi(p)} F_{g}\left(\tau_{1} v-v \frac{\log p}{\log X}\right)+o(1)\right) .
$$

Passing from this sum to the stated integral is a standard exercise in Riemann-Stieltjes integration, or summation by parts. For example, we may write the sum as

$$
\int_{z^{-}}^{s}\left(1-\frac{\log T}{\log y}\right) F_{g}\left(\tau_{1} v-v \frac{\log T}{\log X}\right) \frac{d S(T)}{\log T}
$$

with

$$
S(T)=\sum_{p \leqslant T} \frac{\rho_{1}(p)}{\phi(p)} \log p .
$$

If $z=X^{1 / v}, s=X^{1 / w}, y=X^{1 / u}$, then (7) implies that the integral in (31) is asymptotic to

$$
g \int_{X^{1 / v}}^{X^{1 / w}}\left(1-\frac{\log T}{\log X^{1 / u}}\right) F_{g}\left(v\left(\tau_{1}-\frac{\log T}{\log X}\right)\right) \frac{d \log T}{\log T},
$$

Performing the change of variables $T=X^{1 / t}$ finishes the proof.

Lemma 6.3. Let $z=X^{1 / v}, s=X^{1 / w}, y=X^{1 / u}$ where $0<\frac{1}{v}<\frac{1}{w}<\tau_{1} \leqslant \frac{1}{2}<\frac{1}{u}<\tau_{2} \leqslant 1$.

$$
S_{2} \leqslant X V(z) \frac{g v}{e^{\gamma}}\left\{\int_{u}^{w}\left(1-\frac{u}{t}\right) F_{g+1}\left(v\left(\tau_{2}-\frac{1}{t}\right)\right) \frac{d t}{t}+o(1)\right\} .
$$

Proof. Here we use (11) to swap $S\left(A_{p}, z\right)$ for $S\left(A^{\prime}, z\right)$, since

$$
S\left(A_{p}, z\right) \leqslant S\left(A^{\prime}, z\right),
$$


and then apply the $(g+1)$-dimensional upper bound DHR sieve in (19) to $S\left(A^{\prime}, z\right)$, with $X$ replaced by $X^{\prime}, V(z)$ replaced by $V^{\prime}(z), z=X^{1 / v}$, and $y=X^{\tau_{2}}(\log X)^{-B^{\prime}} / p$ for a suitably large $B^{\prime}$. Using (18) to control the remainder term gives

$$
S\left(A^{\prime}, z\right) \leqslant X^{\prime} V^{\prime}(z)\left(F_{g+1}\left(\frac{\log \left(X^{\tau_{2}}(\log X)^{-B^{\prime}} / p\right)}{\log X^{1 / v}}\right)+o(1)\right) .
$$

Appealing to (24) to perturb the argument of $F_{g+1}$ so that

$$
F_{g+1}\left(\frac{\log \left(X^{\tau_{2}} / p\right)}{\log X^{1 / v}}-B^{\prime} v \frac{\log \log X}{\log X}\right) \leqslant F_{g+1}\left(\frac{\log \left(X^{\tau_{2}} / p\right)}{\log X^{1 / v}}\right)+O\left(\frac{\log \log X}{\log X}\right),
$$

gives

$$
S\left(A^{\prime}, z\right) \leqslant X^{\prime} V^{\prime}(z)\left(F_{g+1}\left(\frac{\log \left(X^{\tau_{2}} / p\right)}{\log X^{1 / v}}\right)+o(1)\right) .
$$

Replacing $V^{\prime}(z)$ and $X^{\prime}$ with their corresponding expressions in (16) and (17),

$$
S\left(A^{\prime}, z\right) \leqslant X V(z) \frac{\rho_{1}(p)}{p} e^{-\gamma} \frac{\log X}{\log z}\left(F_{g+1}\left(v \tau_{2}-v \frac{\log p}{\log X}\right)+o(1)\right) .
$$

Summing over $s \leqslant p<y$ in $S_{2}$ then gives

$$
S_{2} \leqslant X V(z) e^{-\gamma} v\left(\sum_{s \leqslant p<y}\left(1-\frac{\log p}{\log y}\right) \frac{\rho_{1}(p)}{p} F_{g+1}\left(v \tau_{2}-v \frac{\log p}{\log X}\right)+o(1)\right),
$$

since (8) implies that

$$
\sum_{s \leqslant p<y} \frac{\rho_{1}(p)}{p} \ll g \log \left(\frac{\log y}{\log s}\right) \ll g \log \frac{w}{u} \ll 1 .
$$

Passing from the sum in (32) to the stated integral is a standard exercise. Note that this sum is

$$
\int_{s^{-}}^{y}\left(1-\frac{\log T}{\log y}\right) F_{g}\left(\tau_{2} v-v \frac{\log T}{\log X}\right) \frac{d S(T)}{\log T},
$$

with

$$
S(T)=\sum_{p \leqslant T} \frac{\rho_{1}(p)}{p} \log p .
$$

Recalling that $s=X^{1 / w}, y=X^{1 / u}$, and using (8), the integral in (33) is asymptotic to

$$
g \int_{X^{1 / w}}^{X^{1 / u}}\left(1-\frac{\log T}{\log X^{1 / u}}\right) F_{g+1}\left(v\left(\tau_{2}-\frac{\log T}{\log X}\right)\right) \frac{d \log T}{\log T},
$$

Performing the change of variables $T=X^{1 / t}$ finishes the proof.

Combining Lemma 6.1, Lemma 6.2, and Lemma 6.3 gives

Lemma 6.4. Let $0<\frac{1}{v}<\frac{1}{w}<\tau_{1} \leqslant \frac{1}{2}<\frac{1}{u}<\tau_{2} \leqslant 1$. Then

$$
W(A) \geqslant\left(\eta f_{g}\left(\tau_{1} v\right)-\left(I(u, w, v)+\frac{v}{e^{\gamma}} J(u, w, v)\right)+o(1)\right) X V(z)
$$

where

$$
I(u, w, v):=g \int_{w}^{v}\left(1-\frac{u}{s}\right) F_{g}\left(v\left(\tau_{1}-\frac{1}{s}\right)\right) \frac{d s}{s},
$$


and,

$$
J(u, w, v):=g \int_{u}^{w}\left(1-\frac{u}{s}\right) F_{g+1}\left(v\left(\tau_{2}-\frac{1}{s}\right)\right) \frac{d s}{s} .
$$

\section{Simple Estimates FOR THE INTEGRALS}

Analysis for higher dimensional sieves is obstructed by the evaluation of $I:=I(u, w, v)$ and $J:=J(u, w, v)$, appearing above in (34) and (35). Useful estimates of these integrals are presented below. Analysis closely follows Section 11.4 of Diamond-Halberstam [4].

Lemma 7.1. Let $\xi_{1}:=v \tau_{1}+1-\frac{v}{w}$, and $0<\frac{1}{v}<\frac{1}{w}<\tau_{1} \leqslant \frac{1}{2}<\frac{1}{u}<\tau_{2} \leqslant 1$. If $\xi_{1} \geqslant \beta_{g}$, then

$$
\frac{1}{f_{g}\left(\tau_{1} v\right)} I \leqslant\left(g+\frac{u}{v} \xi_{1}\left(1-\frac{f_{g}\left(\xi_{1}\right)}{f_{g}\left(\tau_{1} v\right)}\right)\right) \log \frac{v}{w}+\left(1-\frac{f_{g}\left(\xi_{1}\right)}{f_{g}\left(\tau_{1} v\right)}\right) \xi_{1} \frac{w}{v}\left(1-\frac{u}{w}\right)-g\left(\frac{u}{w}-\frac{u}{v}\right) .
$$

Proof. Let $t-1=v\left(\tau_{1}-\frac{1}{s}\right)$, so $s=v /\left(v \tau_{1}+1-t\right)$. Under this change of variables,

$$
I=g \frac{u}{v} \int_{\xi_{1}}^{v \tau_{1}} F_{g}(t-1) \frac{t-\xi_{1}+\frac{v}{u}-\frac{v}{w}}{v \tau_{1}+1-t} d t .
$$

We then separate the integral so that

$$
I=I_{1}+g \frac{u}{v}\left(\frac{v}{u}-\frac{v}{w}\right) I_{2}
$$

where

$$
I_{1}:=g \frac{u}{v} \int_{\xi_{1}}^{v \tau_{1}} F_{g}(t-1) \frac{t-\xi_{1}}{v \tau_{1}+1-t} d t
$$

and

$$
I_{2}:=\int_{\xi_{1}}^{v \tau_{1}} F_{g}(t-1) \frac{d t}{v \tau_{1}+1-t}
$$

Integrating by parts,

$$
\begin{aligned}
I_{1} & =-g \frac{u}{v} \int_{\xi_{1}}^{v \tau_{1}} F_{g}(t-1)\left(t-\xi_{1}\right) d\left(\log \left(v \tau_{1}+1-t\right)\right) \\
& =g \frac{u}{v} \int_{\xi_{1}}^{v \tau_{1}}\left(F_{g}(t-1)+F_{g}^{\prime}(t-1)\left(t-\xi_{1}\right)\right) \log \left(v \tau_{1}+1-t\right) d t \\
& <g \frac{u}{v} \int_{\xi_{1}}^{v \tau_{1}} F_{g}(t-1) \log \left(v \tau_{1}+1-t\right) d t
\end{aligned}
$$

since $F$ is decreasing. Next, if $\xi_{1} \geqslant \beta_{g}$, then $t \geqslant \beta_{g}$, and we can use (22) to observe that

$$
I_{1}<\frac{u}{v} \int_{\xi_{1}}^{v \tau_{1}}\left(t^{g} f_{g}(t)\right)^{\prime} t^{1-g} \log \left(v \tau_{1}+1-t\right) d t .
$$

Integrating by parts, and using the fact that $f$ is increasing, gives

$$
\begin{aligned}
I_{1} & <\frac{u}{v} \int_{\xi_{1}}^{v \tau_{1}} f_{g}(t)\left((g-1) \log \left(v \tau_{1}+1-t\right)+\frac{t}{v \tau_{1}+1-t}\right) d t-\frac{u}{v} \xi_{1} f_{g}\left(\xi_{1}\right) \log \frac{v}{w} \\
& <\frac{u}{v} f_{g}\left(v \tau_{1}\right) \int_{\xi_{1}}^{v \tau_{1}}\left((g-1) \log \left(v \tau_{1}+1-t\right)+\frac{t}{v \tau_{1}+1-t}\right) d t-\frac{u}{v} \xi_{1} f_{g}\left(\xi_{1}\right) \log \frac{v}{w} .
\end{aligned}
$$


The remaining integral is $\frac{v}{w}\left(g+\frac{w}{v} \xi_{1}\right) \log \frac{v}{w}-g\left(\frac{v}{w}-1\right)$, so that

$$
I_{1}<f_{g}\left(v \tau_{1}\right)\left(\frac{u}{w}\left(g+\frac{w}{v} \xi_{1}\right) \log \frac{v}{w}-g\left(\frac{u}{w}-\frac{u}{v}\right)-\frac{u}{v} \xi_{1} \frac{f_{g}\left(\xi_{1}\right)}{f_{g}\left(\tau_{1} v\right)} \log \frac{v}{w}\right) .
$$

For $I_{2}$, we make use of (22) and integrate by parts to observe that

$$
\begin{aligned}
I_{2} & =\int_{\xi_{1}}^{v \tau_{1}}\left(t^{g} f_{g}(t)\right)^{\prime} \frac{d t}{g t^{g-1}\left(v \tau_{1}+1-t\right)} \\
& =\frac{f_{g}\left(v \tau_{1}\right)}{g} v \tau_{1}-\frac{f_{g}\left(\xi_{1}\right)}{g} \xi_{1} \frac{w}{v}+\int_{\xi_{1}}^{v \tau_{1}} f_{g}(t)\left(\frac{g-1}{g\left(v \tau_{1}+1-t\right)}-\frac{t}{g\left(v \tau_{1}+1-t\right)^{2}}\right) d t .
\end{aligned}
$$

Since $f$ is increasing,

$$
I_{2} \leqslant \frac{f_{g}\left(v \tau_{1}\right)}{g}\left(v \tau_{1}-\frac{f_{g}\left(\xi_{1}\right)}{f_{g}\left(\tau_{1} v\right)} \xi_{1} \frac{w}{v}+g \int_{\xi_{1}}^{v \tau_{1}}\left(\frac{g-1}{g\left(v \tau_{1}+1-t\right)}-\frac{t}{g\left(v \tau_{1}+1-t\right)^{2}}\right) d t\right) .
$$

The remaining integral is $\log \frac{v}{w}-\frac{v \tau_{1}+1}{g}\left(1-\frac{w}{v}\right)$, and so

$$
g \frac{u}{v}\left(\frac{v}{u}-\frac{v}{w}\right) I_{2}<f_{g}\left(\tau_{1} v\right)\left(1-\frac{u}{w}\right)\left(\left(1-\frac{f_{g}\left(\xi_{1}\right)}{f_{g}\left(\tau_{1} v\right)}\right) \xi_{1} \frac{w}{v}+g \log \frac{v}{w}\right) .
$$

Inserting the bounds (37) and (38) into (36) gives the stated lemma.

Lemma 7.2. Let $\xi_{2}:=v \tau_{2}+1-\frac{v}{u}$, and $0<\frac{1}{v}<\frac{1}{w}<\tau_{1} \leqslant \frac{1}{2}<\frac{1}{u}<\tau_{2} \leqslant 1$. If $\xi_{2} \geqslant \beta_{g+1}$, then

$$
\frac{v}{e^{\gamma} f_{g}\left(\tau_{1} v\right)} J \leqslant \frac{1}{f_{g}\left(\tau_{1} v\right)} \frac{v}{e^{\gamma}} g\left(\log \frac{w}{u}-1+\frac{u}{w}\right)+\xi_{2} \frac{g}{g+1} \frac{u}{e^{\gamma}} \frac{1-f_{g+1}\left(\xi_{2}\right)}{f_{g}\left(\tau_{1} v\right)} \log \frac{v}{u} .
$$

Proof. Let $t-1=v\left(\tau_{2}-1 / s\right)$, so $s=v /\left(v \tau_{2}+1-t\right)$. Under this change of variables,

$$
\begin{aligned}
J & =g \frac{u}{v} \int_{\xi_{2}}^{v \tau_{2}+1-\frac{v}{w}} F_{g+1}(t-1)\left(\frac{v}{u}-v \tau_{2}-1+t\right) \frac{d t}{v \tau_{2}+1-t} \\
& =-g \frac{u}{v} \int_{\xi_{2}}^{v \tau_{2}+1-\frac{v}{w}} F_{g+1}(t-1)\left(t-\xi_{2}\right) d \log \left(v \tau_{2}+1-t\right) .
\end{aligned}
$$

Integrating by parts, and then using the fact that $F>1$, we have

$$
\begin{array}{r}
J<g \frac{u}{v}\left[\int_{\xi_{2}}^{v \tau_{2}+1-\frac{v}{w}}\left(F_{g+1}(t-1)-F_{g+1}^{\prime}(t-1)\left(t-\xi_{2}\right)\right) \log \left(v \tau_{2}+1-t\right) d t\right. \\
\left.-\left(\frac{v}{u}-\frac{v}{w}\right) \log \frac{v}{w}\right] .
\end{array}
$$

Since $F$ is decreasing, $F^{\prime}<0$, and

$$
J<g \frac{u}{v}\left[J_{1}-\left(\frac{v}{u}-\frac{v}{w}\right) \log \frac{v}{w}\right]
$$

where

$$
J_{1}=\int_{\xi_{2}}^{v \tau_{2}+1-\frac{v}{w}} F_{g+1}(t-1) \log \left(v \tau_{2}+1-t\right) d t .
$$

Next, using (22), and assuming that $\xi_{2} \geqslant \beta_{g+1}$, we rewrite

$$
J_{1}=\int_{\xi_{2}}^{v \tau_{2}+1-\frac{v}{w}}\left(t^{g+1} f_{g+1}(t)\right)^{\prime} \log \left(v \tau_{2}+1-t\right) \frac{d t}{t^{g}(g+1)} .
$$


Integrating by parts, we find that

$$
J_{1}=\frac{\left(v \tau_{2}+1-\frac{v}{w}\right) f_{g+1}\left(v \tau_{2}+1-\frac{v}{w}\right) \log \frac{v}{w}}{(g+1)}-\frac{\xi_{2} f_{g+1}\left(\xi_{2}\right) \log \frac{v}{u}}{(g+1)}+J_{2},
$$

where

$$
J_{2}=-\int_{\xi_{2}}^{v \tau_{2}+1-\frac{v}{w}} t^{g+1} f_{g+1}(t) d\left(\frac{\log \left(v \tau_{2}+1-t\right)}{t^{g}(g+1)}\right) .
$$

Now, since $f<1$,

$$
J_{1}<\frac{\left(v \tau_{2}+1-\frac{v}{w}\right) \log \frac{v}{w}}{(g+1)}-\frac{\xi_{2} f_{g+1}\left(\xi_{2}\right) \log \frac{v}{u}}{(g+1)}+J_{2},
$$

and

$$
\begin{aligned}
J_{2} & =-\int_{\xi_{2}}^{v \tau_{2}+1-\frac{v}{w}} t^{g+1} f_{g+1}(t) d\left(\frac{\log \left(v \tau_{2}+1-t\right)}{t^{g}(g+1)}\right) \\
& =\frac{1}{(g+1)} \int_{\xi_{2}}^{v \tau_{2}+1-\frac{v}{w}} f_{g+1}(t)\left\{g \log \left(v \tau_{2}+1-t\right)+\frac{t}{v \tau_{2}+1-t}\right\} d t \\
& <\frac{1}{(g+1)} \int_{\xi_{2}}^{v \tau_{2}+1-\frac{v}{w}}\left\{g \log \left(v \tau_{2}+1-t\right)+\frac{t}{v \tau_{2}+1-t}\right\} d t .
\end{aligned}
$$

Calculating the remaining integral, we conclude that

$$
J_{2}<\frac{g}{g+1}\left(v \tau_{2}+1-\frac{v}{w}\right) \log \frac{v}{w}-\frac{g}{g+1} \xi_{2} \log \frac{v}{u}+\left(v \tau_{2}+1\right) \log \frac{w}{u}-\frac{v}{u}+\frac{v}{w} .
$$

Combining (41) and (40), we have

$$
\begin{aligned}
& J_{1}<\left(v \tau_{2}+1-\frac{v}{w}\right) \log \frac{v}{w}-\frac{\xi_{2}}{g+1} f_{g+1}\left(\xi_{2}\right) \log \frac{v}{u} \\
&-\frac{g}{g+1} \xi_{2} \log \frac{v}{u}+\left(v \tau_{2}+1\right) \log \frac{w}{u}-\frac{v}{u}+\frac{v}{w} .
\end{aligned}
$$

Since $v \tau_{2}+1-\frac{v}{w}=\left(\frac{v}{u}-\frac{v}{w}\right)+\left(v \tau_{2}+1-\frac{v}{u}\right)=\left(\frac{v}{u}-\frac{v}{w}\right)+\xi_{2}$, we conclude from (39) that

$$
J<g \frac{u}{v}\left(-\frac{\xi_{2}}{g+1} f_{g+1}\left(\xi_{2}\right) \log \frac{v}{u}+\xi_{2} \log \frac{v}{w}-\frac{g}{g+1} \xi_{2} \log \frac{v}{u}+\left(v \tau_{2}+1\right) \log \frac{w}{u}-\frac{v}{u}+\frac{v}{w}\right)
$$

or equivalently,

$$
J<g \frac{u}{v}\left(-\frac{\xi_{2}}{g+1} f_{g+1}\left(\xi_{2}\right) \log \frac{v}{u}+\xi_{2} \log \frac{v}{u}-\frac{g}{g+1} \xi_{2} \log \frac{v}{u}+\frac{v}{u} \log \frac{w}{u}-\frac{v}{u}+\frac{v}{w}\right) .
$$

Simplifying the right-hand side, this reads,

$$
J<\frac{g}{g+1} \frac{u}{v} \xi_{2}\left(1-f_{g+1}\left(\xi_{2}\right)\right) \log \frac{v}{u}+g\left(\log \frac{w}{u}-1+\frac{u}{w}\right) .
$$

Multiplying this inequality by $\frac{v}{e^{\gamma} f_{g}\left(\tau_{1} v\right)}$ gives the stated lemma. 


\section{Proof of TheOREM 1}

Setting $\xi_{1}=\beta_{g}$ in Lemma 7.1, and $\xi_{2}=\beta_{g+1}$ in Lemma 7.2 gives

$$
\frac{1}{f_{g}\left(\tau_{1} v\right)} I \leqslant\left(g+\frac{u}{v} \beta_{g}\right) \log \frac{v}{w}+\frac{w}{v} \beta_{g}\left(1-\frac{u}{w}\right)-g\left(\frac{u}{w}-\frac{u}{v}\right)
$$

and

$$
\frac{v}{e^{\gamma} f_{g}\left(\tau_{1} v\right)} J \leqslant \frac{1}{f_{g}\left(\tau_{1} v\right)}\left(\frac{v}{e^{\gamma}} g\left(\log \frac{w}{u}-1+\frac{u}{w}\right)+\frac{\beta_{g+1}}{g+1} \frac{u g}{e^{\gamma}} \log \frac{v}{u}\right) .
$$

Setting $\tau_{1}=1 / 2$ and $\tau_{2}=1$, this choice of $\xi_{1}$ and $\xi_{2}$ implies that

$$
u=1+\frac{\beta_{g+1}-1}{v-\left(\beta_{g+1}-1\right)},
$$

and

$$
w=2\left(1+\frac{2\left(\beta_{g}-1\right)}{v-2\left(\beta_{g}-1\right)}\right) .
$$

The parameters $u$ and $w$ will therefore be completely determined by our choice of $v$.

To simplify the analysis, we bound the ratio $w / u$, defined for $v>\max \left\{\beta_{g+1}-1,2\left(\beta_{g}-1\right)\right\}$. Let $N \geqslant 3$ be chosen so that $N\left(\beta_{g+1}-1\right)>\max \left\{\beta_{g+1}-1,2\left(\beta_{g}-1\right), 4\left(\beta_{g}-1\right)-\left(\beta_{g+1}-1\right)\right\}$. Assuming that

$$
v \geqslant N\left(\beta_{g+1}-1\right)
$$

then

$$
\frac{4}{3} \leqslant \frac{w}{u} \leqslant 4
$$

The upper bound is easy to see since

$$
\frac{w}{u}=\frac{2\left(v-\left(\beta_{g+1}-1\right)\right)}{v-2\left(\beta_{g}-1\right)} \leqslant 4
$$

if $v \geqslant 4\left(\beta_{g}-1\right)-\left(\beta_{g+1}-1\right)$, which holds for (46). Next, if $\max \left\{\beta_{g+1}-1,2\left(\beta_{g}-1\right)\right\}=\beta_{g+1}-1$,

$$
g(v):=\frac{2\left(v-\left(\beta_{g+1}-1\right)\right)}{v-2\left(\beta_{g}-1\right)}
$$

is an increasing function. Therefore, for $v$ satisfying (46),

$$
g(v) \geqslant g\left(N\left(\beta_{g+1}-1\right)\right)=\frac{2(N-1)\left(\beta_{g+1}-1\right)}{N\left(\beta_{g+1}-1\right)-2\left(\beta_{g}-1\right)} \geqslant \frac{2(N-1)}{N} \geqslant \frac{4}{3},
$$

since $\beta_{g} \geqslant 1$, and $N \geqslant 3$. If, on the other hand, $\max \left\{\beta_{g+1}-1,2\left(\beta_{g}-1\right)\right\}=2\left(\beta_{g}-1\right)$, then

$$
g(v) \geqslant 2 \text {, }
$$

since this is equivalent to $\beta_{g+1}-1 \leqslant 2\left(\beta_{g}-1\right)$. In either case, the lower bound for $\frac{w}{u}=g(v)$ in (47) holds.

Using (47), the bound for $J$ in (43) simplifies to

$$
\begin{aligned}
\frac{v}{e^{\gamma} f_{g}\left(\tau_{1} v\right)} J & \leqslant\left(\frac{v}{e^{\gamma}} g\left(\log 4-1+\frac{3}{4}\right)+\frac{\beta_{g+1}}{g+1} \frac{u g}{e^{\gamma}} \log \frac{v}{u}\right)\left(1+O\left(e^{-v / 2}\right)\right) \\
& \leqslant \frac{v g}{C_{0}}+\frac{\beta_{g+1}}{g+1} \frac{u g}{e^{\gamma}} \log \frac{v}{u}+O\left(\frac{v g}{e^{v / 2}}+\frac{u g \log v}{e^{v / 2}}\right),
\end{aligned}
$$


where we have used the boundary condition in (23), and defined

$$
C_{0}:=\frac{e^{\gamma}}{\log 4-\frac{1}{4}}
$$

Ultimately, our choice of $v$ in (53) will guarantee that the error term above is $o(1)$, and that our assumption that $v \geqslant N\left(\beta_{g+1}-1\right)$ in (46) is valid provided $k$ is sufficiently large, say

$$
k \geqslant \frac{(N-1)^{2}\left(\beta_{g+1}-1\right)}{C_{0}} .
$$

Lemma 5.1, Lemma 6.4, and (9) guarantee (1) is satisfied provided we select an $r$ such that

$$
r>g k u-1+\frac{1}{f_{g}\left(\tau_{1} v\right)} I(u, w, v)+\frac{v}{e^{\gamma} f_{g}\left(\tau_{1} v\right)} J(u, w, v) .
$$

Ignoring error terms, the bounds in (42) and (48) show that it is enough to select an $r$ such that

$$
r>g k u-1+\left(g+\frac{u}{v} \beta_{g}\right) \log \frac{v}{w}+\frac{w}{v} \beta_{g}\left(1-\frac{u}{w}\right)-g\left(\frac{u}{w}-\frac{u}{v}\right)+\frac{v g}{C_{0}}+\frac{\beta_{g+1}}{g+1} \frac{u g}{e^{\gamma}} \log \frac{v}{u} .
$$

In search of the smallest such $r$, we choose $v$ to minimize the expression on the right. For the sake of simplicity, we focus on the most problematic terms in this expression, given by

$$
M(v):=g k u+\frac{v g}{C_{0}}=g k+\frac{\left(\beta_{g+1}-1\right) g k}{v-\left(\beta_{g+1}-1\right)}+\frac{v g}{C_{0}},
$$

where $C_{0}$ is defined in (49), also keeping in mind (44). The minimum is achieved at

$$
v=\beta_{g+1}-1+\sqrt{C_{0}\left(\beta_{g+1}-1\right) k},
$$

at which

$$
M(v)=g k+g k\left(2 \sqrt{\left(\beta_{g+1}-1\right) /\left(C_{0} k\right)}+\left(\beta_{g+1}-1\right) /\left(C_{0} k\right)\right),
$$

and the remaining terms in (52) are $O(g \log g k)$. Therefore, the admissible $r$ in (52) take the form

$$
r>g k+c_{1} g^{3 / 2} k^{1 / 2}+c_{2} g^{2}+O(g \log g k),
$$

where

$$
c_{1}=2 \sqrt{\frac{\beta_{g+1}-1}{C_{0} g}} \quad \text { and } \quad c_{2}=\frac{\beta_{g+1}-1}{C_{0} g} .
$$

Both $c_{1}$ and $c_{2}$ are $O(1)$. Thus, our admissible $r$ take the form stated in (2).

Before moving on, note that we have shown (2) for $k$ satisfying (50), but that we may need an even larger $k$ to guarantee that these admissible $r$ are asymptotically better than those in (3). In fact, the main term in (54) satisfies $M(v)<2 g k$ if

$$
\frac{\beta_{g+1}-1}{C_{0} k}<3-2 \sqrt{2} \text {. }
$$

Therefore, we suppose that

$$
k>\max \left\{\frac{(N-1)^{2}\left(\beta_{g+1}-1\right)}{C_{0}}, \frac{\beta_{g+1}-1}{C_{0}(3-2 \sqrt{2})}\right\} .
$$

However, numerical data suggests that the improvements appear much earlier.

For the admissible $r$-values in Table 2, we briefly describe our choices of $v, w$, and $u$, for each fixed $g$ and $k$. All numerical experiments were conducted using W. Galway's Mathematica 
package [5]. We chose the parameter $v$ to be of the form $v=\alpha_{g}+n$, where $n$ is a positive integer. Next, we chose $w$ to minimize the expression on the right in (51), which amounts to solving

$$
F_{g}\left(v\left(\frac{1}{2}-\frac{1}{w}\right)\right)-\frac{v}{e^{\gamma}} F_{g+1}\left(v\left(1-\frac{1}{w}\right)\right)=0 .
$$

With these choices of $v$ and $w$, we then chose $u$ to minimize the expression in (51) by solving

$$
k f_{g}\left(\frac{v}{2}\right)-\int_{w}^{v} F_{g}\left(v\left(\frac{1}{2}-\frac{1}{s}\right)\right) \frac{d s}{s^{2}}-\frac{v}{e^{\gamma}} \int_{w}^{u} F_{g+1}\left(v\left(1-\frac{1}{s}\right)\right) \frac{d s}{s^{2}}=0 .
$$

This process was repeated for many values of $n$ to arrive at the stated admissible $r$-values.

\section{CONCLUDING REMARKS}

More general results are readily available. For example, one could consider polynomials $H$ whose irreducible components have different degrees. In addition, the work of Booker and Browning [2] allows one to capture squarefree values, rather than almost-primes, if these irreducible components have degree 3 or less. The polynomial sequence considered here was chosen mainly for illustrative purposes.

\section{REFERENCES}

[1] N. C. Ankeny and H. Onishi. The general sieve. Acta Arith, 10:31-62, 1964/1965.

[2] A. R. Booker and T. D. Browning. Square-free values of reducible polynomials. Discrete Anal., Paper No. 8, 16, 2016.

[3] H. G. Diamond and H. Halberstam. On the sieve parameters $\alpha_{\kappa}$ and $\beta_{\kappa}$ for large $\kappa$. J. Number Theory, 67 (1): 52-84, 1997.

[4] H. G. Diamond and H. Halberstam. A higher-dimensional sieve method, volume 177 of Cambridge Tracts in Mathematics. Cambridge University Press, Cambridge, 2008. With a appendix by William F. Galway.

[5] W. Galway. Mathematica Package for Sieve Calculations: SieveFunctions.m (version 0.9). https://faculty.math.illinois.edu/SieveTheoryBook/SieveFunctions.m

[6] H. Halberstam and H.-E. Richert. Sieve methods. Academic Press, London-New York, 1974. London Mathematical Society Monographs, No. 4.

[7] A. J. Irving. Almost-prime values of polynomials at prime arguments. Bull. Lond. Math. Soc. 47 (4): 593-606, 2015.

[8] P.-H. Kao, Almost-prime values of polynomials at prime arguments. J. Number Theory, 184: 85-106, 2018.

[9] H.E. Richert. Selberg's sieve with weights. Mathematika, 16, 1-22, 1969.

[10] J. Wu and P. Xi. Quadratic polynomials at prime arguments. Math. Z., 285 (1-2): 631-646, 2017.

Department of Mathematics, The Ohio State University

E-mail address: franze.3@osu.edu

Department of Mathematics and Technology, Flagler College

E-mail address: ckao@flagler.edu 